\title{
Transitional separation bubbles and unsteady aspects of aerofoil stall
}

\author{
N. D. Sandham \\ Aerodynamics and Flight Mechanics Research Group \\ School of Engineering Sciences \\ University of Southampton, \\ Southampton, UK
}

\begin{abstract}
A time-accurate solution method for the coupled potential flow and integral boundary-layer equations is used to study aerofoils near stall, where laboratory experiments have shown high-amplitude lowfrequency oscillations. The laminar-turbulent transition model incorporates an absolute instability formulation, which allows the transition process in separation bubbles to be sustained in the absence of upstream disturbances, in agreement with recent direct numerical simulations. The method is demonstrated to capture large scale flow oscillations with Strouhal numbers and amplitudes comparable to experiments. The success of this particular physical model suggests that bubble bursting is primarily due to a potentialflow/boundary-layer interaction effect, in which relatively simple models of boundary layer transition and turbulence suffice to describe the key phenomena.
\end{abstract}

\section{NOMENCLATURE}

$\begin{array}{ll}a & \text { transition coefficient } \\ B & \text { generic right hand side term } \\ c & \text { aerofoil chord } \\ C_{f} & \text { skin friction } \\ C_{L} & \text { coefficient of lift } \\ C_{p} & \text { coefficient of pressure } \\ E & \text { entrainment } \\ f & \text { frequency; function } \\ H & \text { shape factor } \\ H_{1} & \text { alternate shape parameter }\left(\delta-\delta^{*}\right) / \theta\end{array}$

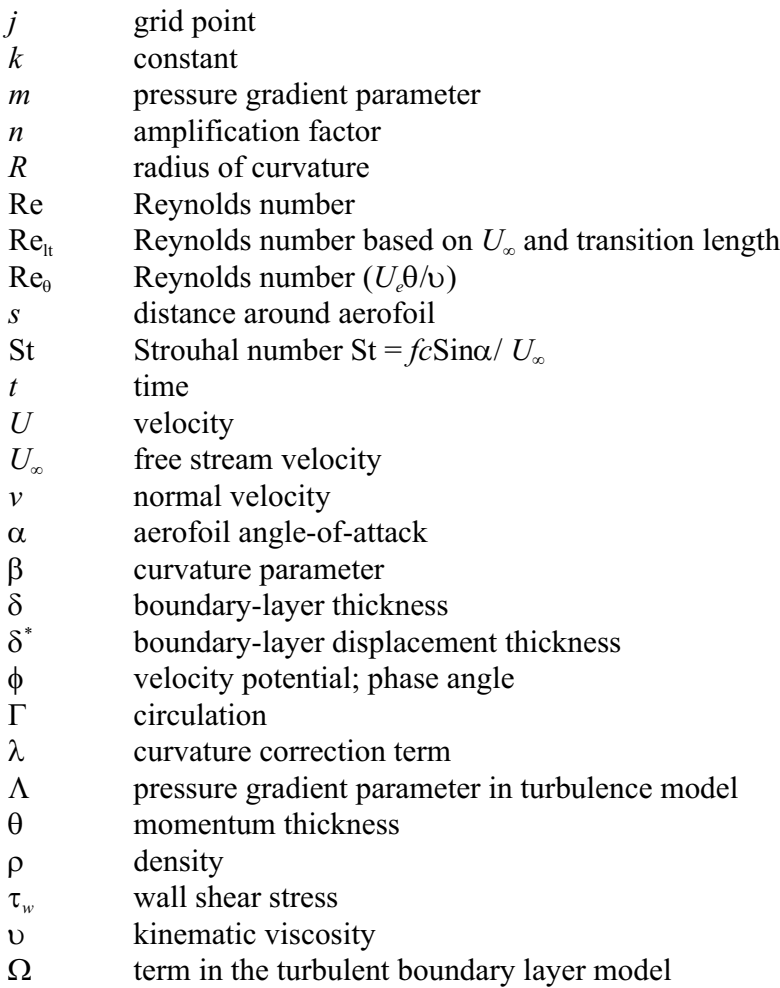




$\begin{array}{ll}\text { Subscripts } \\ \text { crit } & \text { critical } \\ e & \text { edge of boundary layer } \\ e q & \text { equilibrium } \\ E & \text { entrainment equation } \\ H & \text { shape factor equation } \\ n & \text { amplification factor equation } \\ \text { RMS } & \text { root-mean-square } \\ w & \text { wall }\end{array}$

\subsection{INTRODUCTION}

Separation bubbles are important due to the controlling influence that they can have on the overall performance of aerofoils. Leading edge separation bubbles do not greatly influence the pressure distribution but effectively fix the location of transition to turbulence. Additionally, short bubbles may undergo a bursting process which fixes the maximum lift that can be generated by an aerofoil. Since the early observations of Jones ${ }^{(1)}$, much work has been done to study transitional separation bubbles in the laboratory and more recently by direct numerical simulation, with a result that much of the flow physics has been identified, if not yet fully incorporated into prediction methods. Particularly noteworthy in the context of the present journal issue is the contribution from Queen Mary, University of London, including the pioneering experiments covered in the PhD theses of McGregor ${ }^{(2)}$, Gaster $^{(3)}$, Horton ${ }^{(4)}$, Woodward ${ }^{(5)}$ and the more recent direct numerical simulations (DNS) of Alam ${ }^{(6)}$. Some of the early work was reviewed by Young and Horton ${ }^{(7)}$. Figure 1 shows a schematic from Horton ${ }^{(4)}$, which illustrates the main features of a transitional separation bubble. After laminar boundary layer separation a highly unstable detached shear layer forms and transition to turbulence takes place in the detached shear layer. The enhanced momentum transport in the turbulent flow usually enables reattachment and a turbulent boundary layer develops downstream. In the time-averaged picture there is a 'deadair' region under the detached shear layer immediately after separation and a strong recirculation zone near the rear of the bubble.

Semi-empirical models (see e.g. Horton ${ }^{(8)}$, Roberts ${ }^{(9)}$, Weibust ${ }^{(10)}$ ) and integral boundary-layer computational methods (Drela and Giles $^{(11)}$ ) do a good job of collapsing data from laboratory experiments and enable predictions of bubble behaviour up to bursting. In these models the bubble is split into two parts: the transitional part from laminar separation up to transition and the turbulent part from transition to reattachment. Rist and co-workers ${ }^{(12,13)}$ have made extensive investigations of transition in separation bubbles, while Alam and Sandham ${ }^{(14)}$ and Spalart and Strelets ${ }^{(15)}$ carried out the first direct numerical simulations of the turbulent re-attachment and downstream recovery after transition. Some aspects of the transition process are becoming clearer. Time-averaged bubble velocity profiles are locally convectively unstable if the amount of reverse flow is small, but become absolutely unstable for reverse-flow levels greater than $15-20 \%$ of the local free stream velocity. There is also a possibility of vortex shedding from bubbles, as seen in the twodimensional simulations of Pauley et $a l^{(16)}$. Bursting itself is less well understood, although Gaster ${ }^{(17)}$ speculated a mechanism whereby the bubble as a whole becomes unstable: above a certain critical condition, a small increase in bubble length changes the potential flow such that the bubble lengthens, reinforcing the perturbation and leading to instability. This observation is directly relevant to the current study since it suggests that a model based only on unsteady boundary layer displacement coupled to the potential flow should be able to predict the phenomenon.

Large-amplitude low-frequency oscillations during stall were originally commented on by Jones ${ }^{(1)}$ and interest in this aspect of stall was revived by the work of Zaman et al ${ }^{(18)}$, whose experimental work documented a phenomenon of flow oscillations with Strouhal number (defined as $\mathrm{St}=f_{c} \mathrm{Sin} \alpha / U_{\infty}$ ) St $\approx 0.02$, occurring near the

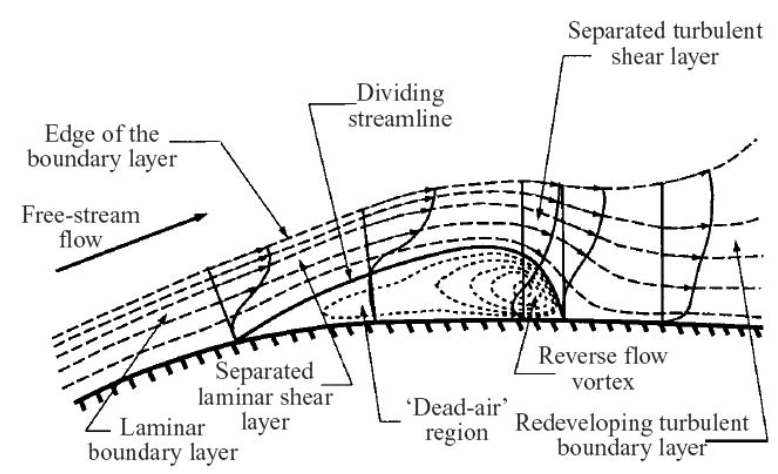

Figure 1. The mean flow structure of a laminar separation bubble (Horton 1968).

maximum lift of an LRN-1007 aerofoil at low Reynolds numbers 1.5 $\times 10^{5}<\operatorname{Re}<3.0 \times 10^{5}$. The oscillations were not connected to wake vortex shedding, which was observed to occur at higher $S t \approx 0 \cdot 2$, nor were any extraneous influences such as structural vibrations to blame, and similar results were observed for two different aerofoil models. As pointed out by Zaman et al, the occurrence of such oscillations is of practical importance, being probably connected to the aeroelastic phenomenon of stall flutter. Follow up experimental work by Bragg et $a l^{(19)}$ extended the results of Zaman for the same aerofoil up to $\operatorname{Re}=1.3 \times 10^{6}$, showing that this was not just a very low Reynolds number phenomenon. Broeren and $\mathrm{Bragg}^{(20)}$ identified the laminar separation bubble as a key aspect of the phenomenon and mapped out the location of boundary layer separation and reattachment points during the oscillation. This showed distinct phases of the oscillation, one involving bubble growth, one involving fully stalled flow and another with a rearwards-moving turbulent separation point. They also demonstrated experimentally that the low frequency oscillation is fundamentally a two-dimensional mode and non-hysteretic in character. This is in contrast with the leading-edge stall found on the NACA2414 aerofoil, for which hysteresis and a three-dimensional 'stall-cell' structure was observed. A further demonstration of the phenomenon is contained in the work of Rinoie and Takemura ${ }^{(21)}$ for the NACA0012 aerofoil at $\mathrm{Re}=130,000$. This investigation included flow visualisations and phase-averaged velocity measurements, demonstrating the fundamental differences between the time averaged flow structure and the periodic bursting cycle, in which the flow was observed to alternate between a short leading edge bubble and fully stalled flow.

In this paper we shall attempt to demonstrate that unsteady bubble bursting and the phenomenon of low-frequency oscillations on aerofoils are predictable by relatively simple methods, albeit methods that include realistic depictions of laminar and turbulent boundary layer flow, including moderately separated flow regions and laminar-turbulent transition. The deliberate simplicity of the model will help to identify which of the many fluid-dynamical features potentially active in separation bubbles are relevant to bubble bursting and the low-frequency mode observed in experiments.

\subsection{COMPUTATIONAL MODEL}

The level of modelling employed in the present work is the same as that employed in the popular aerofoil analysis and design tool XFOIL ${ }^{(22)}$, modelling aspects of which are described in the paper by Drela and Giles ${ }^{(11)}$. A panel method is coupled with a solution of the integral boundary layer equations, including models for laminar and turbulent flow and including a transition model. In the present work 
the method is extended to unsteady flow and to allow for transition via an absolute instability mechanism. All models and all calculations are given in dimensionless form using aerofoil chord and free stream properties as reference quantities. Incompressible flow is assumed.

\subsection{Boundary-layer model}

The basis for the model is the unsteady momentum integral equation

$\frac{\partial U_{e} \delta^{*}}{\partial t}+\frac{\partial U_{e}^{2} \theta}{\partial s}+U_{e} \delta^{*} \frac{\partial U_{e}}{\partial s}=\frac{\tau_{w}}{\rho}$

which relates boundary-layer displacement and momentum thicknesses $\delta^{*}$ and $\theta$ to the local free stream velocity $U_{e}$ and shear stress $\tau_{w}$ variations along the aerofoil surface co-ordinate $s$. This equation appears already in full unsteady form in e.g. Rosenhead ${ }^{(23)}$, but has usually been applied only to steady flow. To this we need to add an interaction condition, giving the surface transpiration velocity $v_{s}$ in an equivalent potential flow with no boundary layer

$v_{s}=\frac{\mathrm{d}\left(U_{e} \delta^{*}\right)}{\mathrm{d} s}$

It should be noted that the time-dependent variable in Equation (1) can be inserted directly into Equation (2) for the transpiration velocity. The potential flow can then be solved using surface transpiration as a boundary condition.

Closure of the governing Equation (1) is obtained via models for the shape factor $H=\delta^{*} / \theta$, amplification factor $n$ and entrainment $E$, which are formulated as transport equations

$\frac{\partial H}{\partial t}+U_{H} \frac{\partial H}{\partial s}=B_{H}$

$\frac{\partial n}{\partial t}+U_{n} \frac{\partial n}{\partial s}=B_{n}$

$\frac{\partial E}{\partial t}+U_{E} \frac{\partial E}{\partial s}=B_{E}$

Here $U_{H} U_{N}$ and $U_{E}$ are convection velocities and the right hand side terms $B_{H} B_{n}$ and $B_{E}$ require modelling. The entrainment equation is only needed when the flow is turbulent, which occurs when $n$ exceeds a critical value $n_{\text {crit }}$. The remaining shear stress term in Equation (1) is determined explicitly in terms of $H$ and $E$.

Models are required for laminar and turbulent boundary layers and for the growth of amplification factor $n$ due to boundary-layer instabilities. Generally, well-known models have been chosen (see Appendix A for details). Where an appropriate model is not directly applicable in the desired numerical framework, as is the case for the laminar boundary layer, the new model has been tuned to match the characteristics of a well-known model. The main novelty relates to the transition modelling, which needs to be updated to take into account recent findings. Direct numerical simulations by Jones et $a l^{(24)}$ have been carried out for a NACA0012 aerofoil at $\alpha=5^{\circ}$. Initially the simulations were forced, but it was observed that when the upstream disturbances were removed, the transition to turbulence was sustained. The mechanism for this phenomenon was found to be an absolute instability of the vortex shedding, in which braid-region disturbances were amplified before being convected upstream. This effect of self-sustained transition, which is not included in the Drela and Giles ${ }^{(11)}$ transition scheme, may be modelled by changing the sign of $U_{n}$ as the shape factor (which is assumed to be related to strength of backflow) increases. A suitable model form is given by

$U_{n}=U_{e}(0 \cdot 1+0 \cdot 2 \operatorname{Tanh}(7-H))$

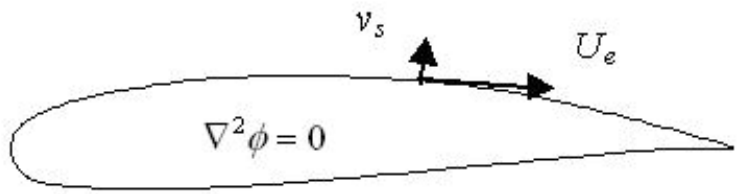

Figure 2. Sketch of aerofoil and auxiliary vortex.

We denote the original model (see Appendix A.2, with $U_{n}=0.3 U_{e}$ ) as the convective instability (CI) model and the new model Equation (6) as the absolute instability (AI) model. The effects on separation bubbles will be studied in Section 3.1. The AI model ensures that bubbles with a strong enough recirculation (corresponding to a high shape factor) will always undergo transition to turbulence, regardless of the level of upstream turbulence.

\subsection{Potential flow, wake model and overall solution methodology}

The potential flow is solved using a constant strength source/doublet panel method (see e.g. Katz and Plotkin ${ }^{(25)}$ ) with Dirichlet boundary conditions for the velocity potential $\phi$. The unsteady wake is modelled with an auxiliary vortex located half a chord downstream of the aerofoil trailing edge (Fig. 2). The time evolution of the auxiliary wake vortex circulation is computed from

$\frac{\mathrm{d} \Gamma_{w}}{\mathrm{~d} t}=\frac{\mathrm{d} \Gamma}{\mathrm{d} t}-k \frac{U_{\infty} \Gamma_{w}}{c}$

For steady flow the second term on the right hand side ensures that the circulation of the auxiliary vortex will decay to zero and the potential flow will reduce to the usual steady flow solution. However, as the aerofoil circulation $\Gamma$ changes, the auxiliary vortex will be activated. The constants $k=0.5$ and the location of the auxiliary vortex were chosen with reference to test calculations for the starting vortex problem ${ }^{(26)}$.

Solution variables $U_{e} \delta^{*}, H, n$ and $E$ are stored at grid points $j$, the first and last of which are at the trailing edge of the aerofoil. The grid points correspond to the panel end points. The boundary layer displacement effect is modelled with additional sources, with strength given by Equation (2), which is differentiated centrally. The output of the panel method is a velocity potential $\phi$ at each panel centre $j+1 / 2$, which is again differentiated centrally to give velocity $U_{e}$ at the original grid points $j$. The trailing-edge velocity is found by averaging the upper and lower surface values from the points nearest to the trailing edge. The final part of the solution vector is the circulation of the auxiliary vortex $\Gamma_{w}$.

The panel method is only of first order overall accuracy so there is probably little to be gained from higher order spatial schemes in evaluating the derivatives in Equations (1) and (3-4). We choose therefore a simple first order upwind scheme, with biasing in the direction of the oncoming $U_{e}$. Spatial discretisation then leads to a system of ordinary differential equations for the solution vector at each grid point, which is advanced in time using a second order Runge-Kutta method with a fixed time step. A numerical limiter has been applied to the rate of change of shape factor by restricting

$\left|\frac{\mathrm{d} H}{\mathrm{~d} t}\right| \leq 0 \cdot 04 \frac{\left|U_{e}\right|}{\theta}$

which is useful in keeping the solution bounded during rapid transients. 
Table 1

List of the aerofoils studied

$\begin{array}{lllll}\text { Case } & \text { Aerofoil } & \text { Re } & \text { Panels } & \begin{array}{c}\text { Reference } \\ \text { Drela \& Giles }\end{array} \\ 1 & \text { LNV109A } & 5.0 \times 10^{5} & 140 & \text { Jones et al }^{(24)} \\ 2 & \text { NACA 0012 } & 0.5 \times 10^{5} & 120 & \text { Rinoie \& Takemura } \\ 3 & \text { NACA 0012 } & 1.3 \times 10^{5} & 120 & \text { Broeren \& Bragg }^{(20)} \\ 4 & \text { Eppler E374 } & 3.0 \times 10^{5} & 120 & \text { Broeren \& Bragg }^{(20)}\end{array}$

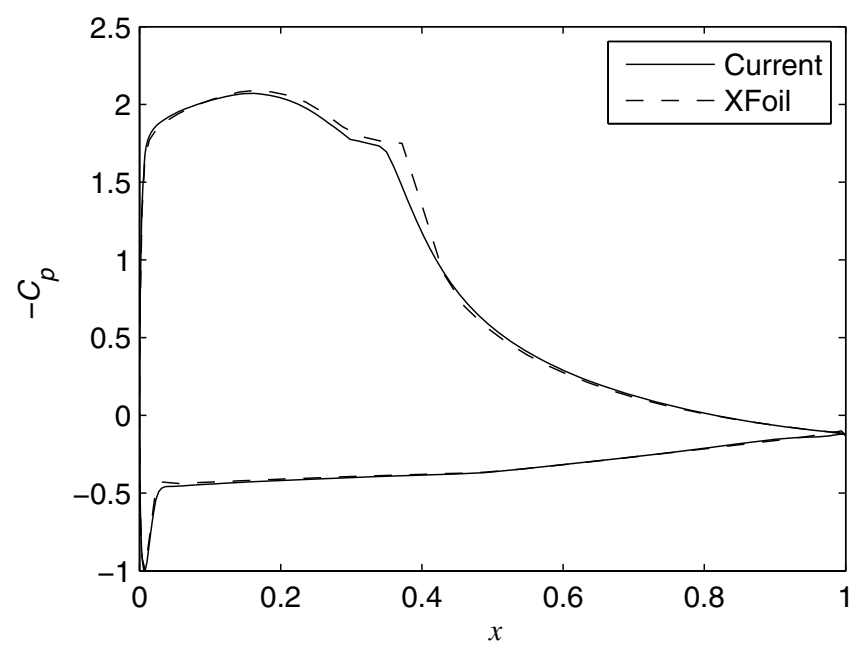

Figure 3. Comparison of pressure distribution at $C_{L}=1.234$ with XFOIL for the LNV109A aerofoil at $\operatorname{Re}=5.0 \times 10^{5}$ (compare with Fig. 7 of Drela and Giles ${ }^{(11)}$ ).

\subsection{RESULTS}

Table 1 lists the five aerofoils selected for study, all of which exhibit transitional separation bubbles during some part of the incidence range from zero to stall. Case 1 is for validation of the pressure distribution for an aerofoil with a short mid-chord separation bubble. Case 2, which has a large separation bubble, is used for a study of the transition modelling. Cases 3 and 4 have experimentallydocumented low-frequency oscillations. By contrast, Case 5 is known to have a sudden leading edge stall.

Unless otherwise stated we take the critical value of the amplification factor to be $n_{\text {crit }}=9$ and $U_{H}=U_{E}=0 \cdot 3 U_{e}$. The calculations are started in each case from zero flow and the freestream velocity is ramped up rapidly to $U_{\infty}=1$. The calculations are then run for at least 20 time units to allow transient effects to disappear before accumulating statistical data. For the NACA aerofoils, the final coefficient in the thickness polynomial ${ }^{(27)}$ was modified to give an identically zero trailing edge thickness. Sensitivity of the results to the number of panels used will be discussed later.

\subsection{Pre-stall calculations}

The LNV109A aerofoil is used as a basic test case for a separation bubble on an aerofoil. The computed pressure distribution at $C_{L}=$ 1.234 is compared on Fig. 3 with results from XFOIL The location of the bubble and the pressure inside the bubble are correctly captured, also with reference to the available experimental data ${ }^{(11)}$.

Case 2, with incidence set to $\alpha=5^{\circ}$, is used to study the effect of the CI and AI variants of the transition model. Figure 4 shows the Reynolds number based on the transition length (i.e. the distance along the aerofoil surface from separation to transition) as a function of $n_{\text {crit }}$, where high values of $n_{\text {crit }}$ correspond to low levels of free stream turbulence. When the original CI model ${ }^{(11)}$ is used there is a

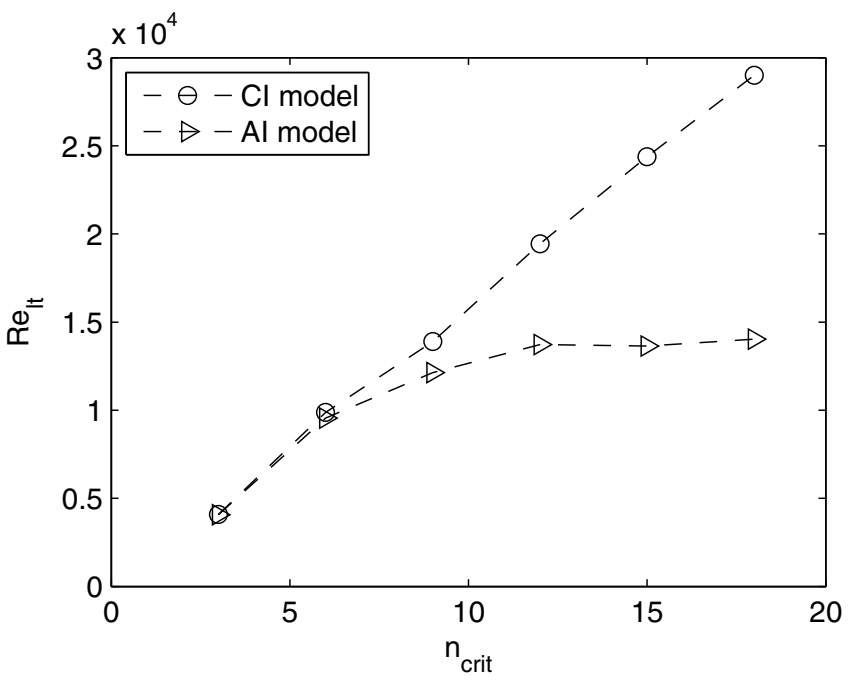

Figure 4. Transition length Reynolds number as a function of critical $n$-factor for a NACA0012 aerofoil at $\operatorname{Re}=50,000$, comparing the convective instability $(\mathrm{Cl})$ model with the modified absolute instability $(\mathrm{Al})$ version.

linear increase of bubble transition length with $n_{\text {crit }}$ and eventually the bubble occupies the whole aerofoil. This is unphysical, as compared with the direct numerical simulations of Jones et $a l^{(24)}$. The AI model variant behaves the same as the CI variant for low values of $n_{\text {crit }}$, corresponding to high levels of free stream turbulence, but tends to a constant transition length as the turbulence level is reduced to zero.

When the time history of lift coefficient for Case 2 is plotted (see Fig. 5), a low amplitude variation is observed (with $C_{L, R M S}=0.04$ ). The frequency corresponds to $S t \approx 0.2$ and is comparable to the vortex shedding seen behind the separation bubble in the DNS. The corresponding $C_{p}$ distributions shown on Fig. 5(b) have undulations that convect over the upper surface of the aerofoil. Such shedding has also been seen in calculations of aerofoils using a Reynoldsaveraged formulation ${ }^{(28)}$. This low amplitude shedding is not central to the present paper, but it is interesting that it appears in the current calculations with no more than an integral boundary layer model. This suggests that the shedding mechanism may be primarily a viscous-inviscid interaction effect. In the present model the KelvinHelmholtz instability, to which shedding is commonly attributed ${ }^{(16,28)}$, is implicit within the transition model, whereas the shedding seems to develop from the rear of the bubble where the flow is treated as turbulent.

Numerical resolution was tested for the NACA aerofoils. At $\alpha=$ $10^{\circ}$ for the NACA2414 halving the number of panels from 120 to 80 changed the lift coefficient from 1.130 to 1.136 , while for the NACA0012 at changing the number of panels from 120 to 80 changed the lift coefficient from 0.819 to 0.826 . From this and other checks we estimate that errors in the lift coefficient due to truncation errors are of the order of $1-2 \%$.

\subsection{Mean and root-mean-square lift coefficient}

The variation with incidence of lift coefficient $C_{L}$ (circles) and rootmean-square lift coefficient $C_{L, R M S}$ (triangles) are shown on Fig. 6(a-c) for Cases 3-5 respectively. Case 3 corresponds to the Rinoie and Takemura $^{(21)}$ configuration. They observed a short separation bubble at $\alpha=10^{\circ}$, but low-frequency oscillations at $\alpha=11 \cdot 5^{\circ}$. The current simulations are in broad agreement. $C_{L}$ (which wasn't reported in the experiments) reaches a maximum for $\alpha=10^{\circ}$ and then reduces rapidly. Oscillations appear first at $\alpha=10 \cdot 75^{\circ}$ and continue to $\alpha=$ 


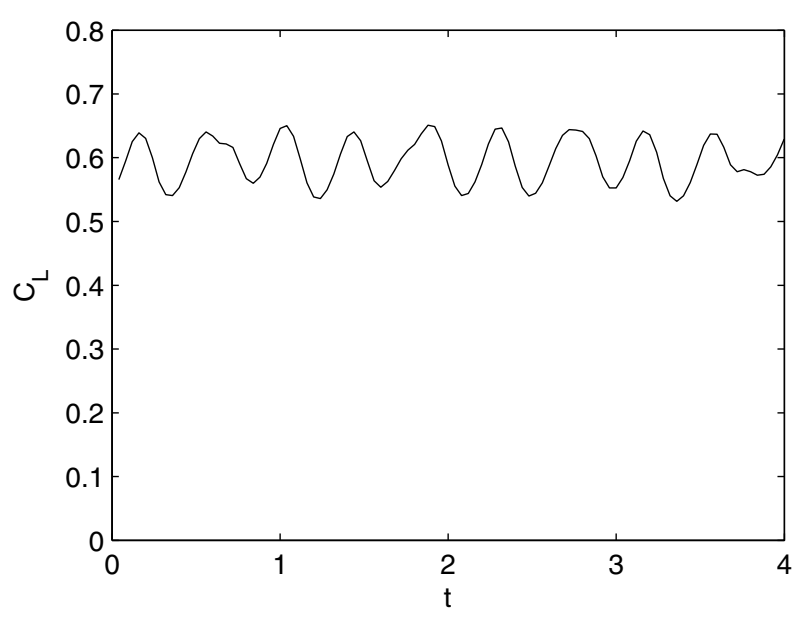

(a)

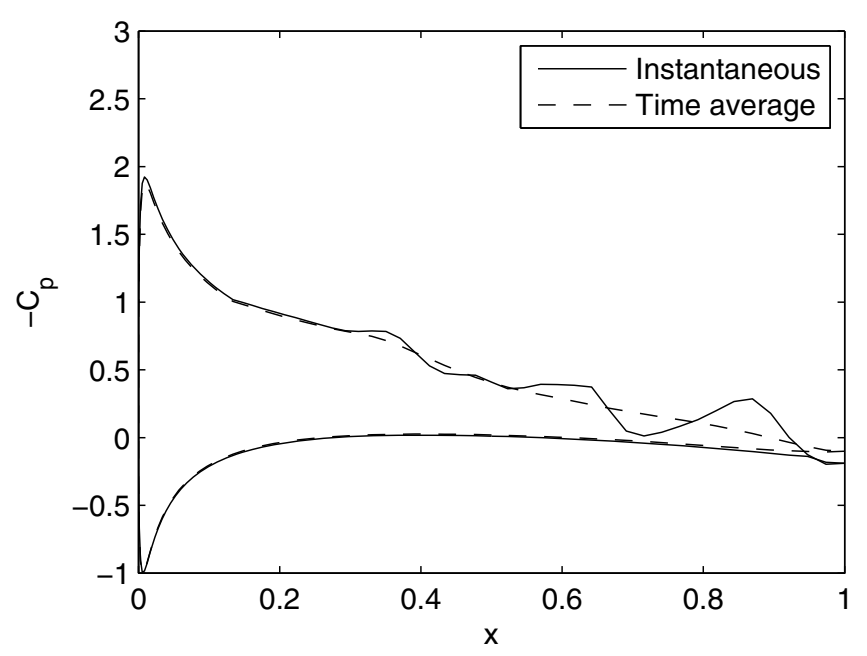

(b)

Figure 5. Time-dependent 'shedding' behaviour observed in calculation of a NACA0012 at $\alpha=5^{\circ}$ and $\operatorname{Re}=50,000$ : (a) variation of lift coefficient with time, and (b) instantaneous pressure coefficient $C_{p}$.

$11 \cdot 75^{\circ}$, by which time $C_{L}$ has reduced to half its maximum value. The maximum $C_{L, R M S}$ is 0.18 , which corresponds to severe oscillations. Other features of this case are typical for the thin-aerofoil type of stall. The separation bubble is susceptible to shedding at low incidences (i.e. $0^{\circ}<\alpha<2^{\circ}$ ), but becomes steady as the bubble length reduces and the bubble itself moves towards the leading edge. The lift slope is seen to reduce as the incidence increases, which is typical of aerofoils with separation bubbles. At $\alpha=10^{\circ}$ the computed bubble length is 0.097 , which is comparable to the experimental value of 0.091. Reducing the number of panels from 120 to 80 at $\alpha=11^{\circ}$ changed $C_{L}$ from 0.747 to 0.736 and $C_{L, R M S}$ from 0.18 to 0.22 .

A similar picture is obtained for the E374 aerofoil, shown on Fig. 6(b). The lift slope shows a marked change at $\alpha=10^{\circ}$ when the separation bubble forms. At $\alpha=10^{\circ}$, just before the start of low frequency oscillations, $C_{L}$ is 1.03 , compared to the experimental value of $1.09^{(20)}$. Compared to the experiment the range of incidence over which oscillations in lift are observed is similar $\left(12.5^{\circ} \leq \alpha \leq\right.$ $14.5^{\circ}$ in model, compared to $12^{\circ} \leq \alpha \leq 14^{\circ}$ in the experiment) and the $C_{L, R M S}$ values are similar $(0.24$ in the computations compared with 0.16 in the experiments).

For the NACA2414 aerofoil (Fig. 6(c)) the model predicts a change in lift slope at $\alpha=5^{\circ}$ (compared to $\alpha=4^{\circ}$ in the experiment),

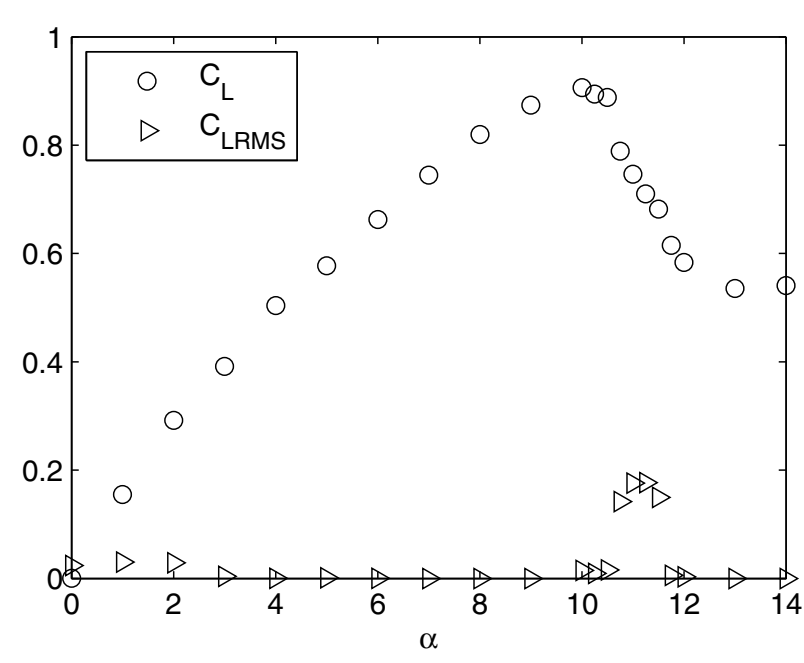

(a)

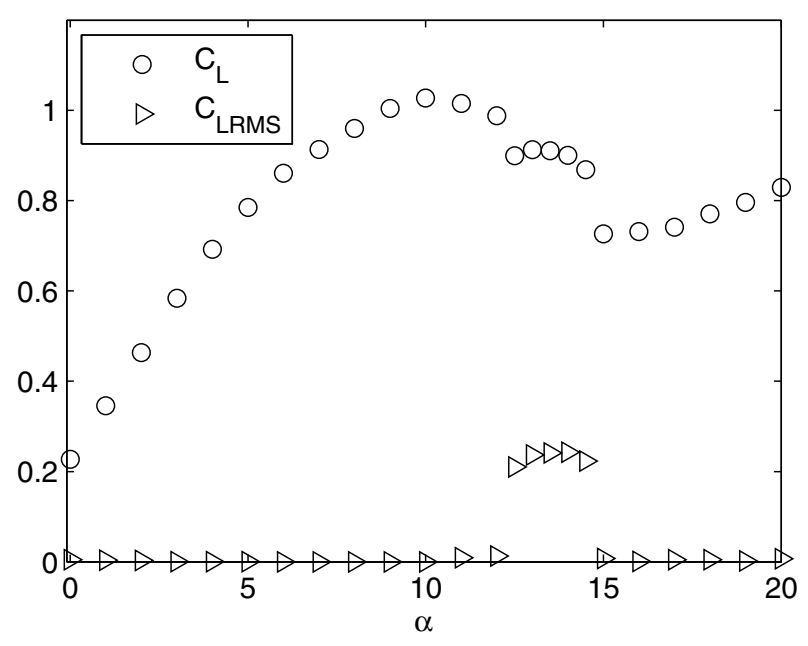

(b)

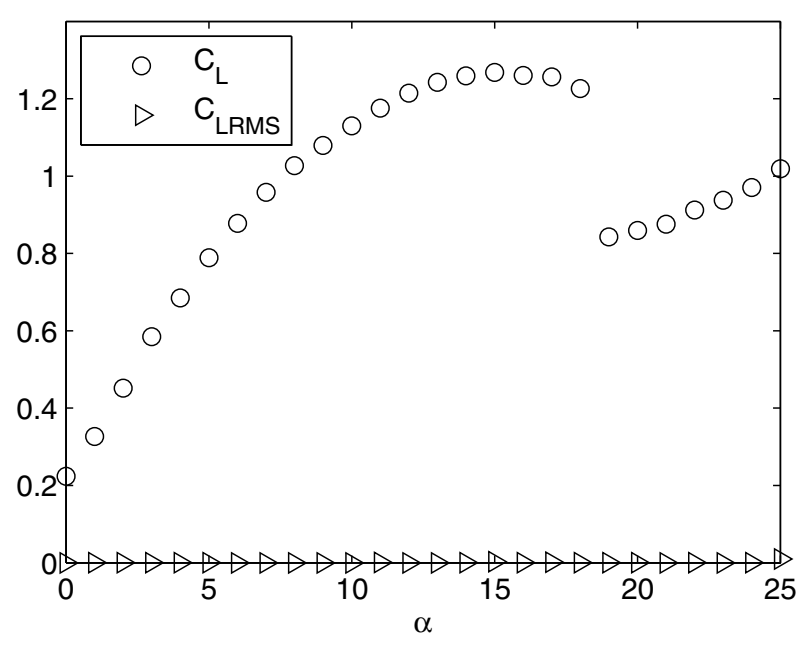

(c)

Figure 6 Lift coefficient and RMS lift coefficient as a function of angle-of-attack for (a) NACA0012 at $\operatorname{Re}=130,000$ (b) E374 at $\mathrm{Re}=300,000$ and (c) NACA2414 at $\operatorname{Re}=300,000$. 


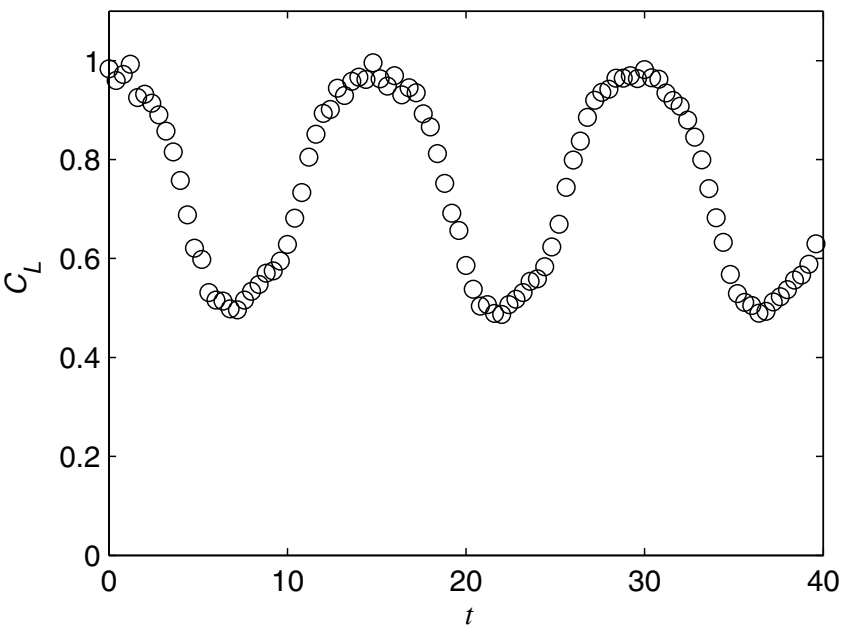

(a)

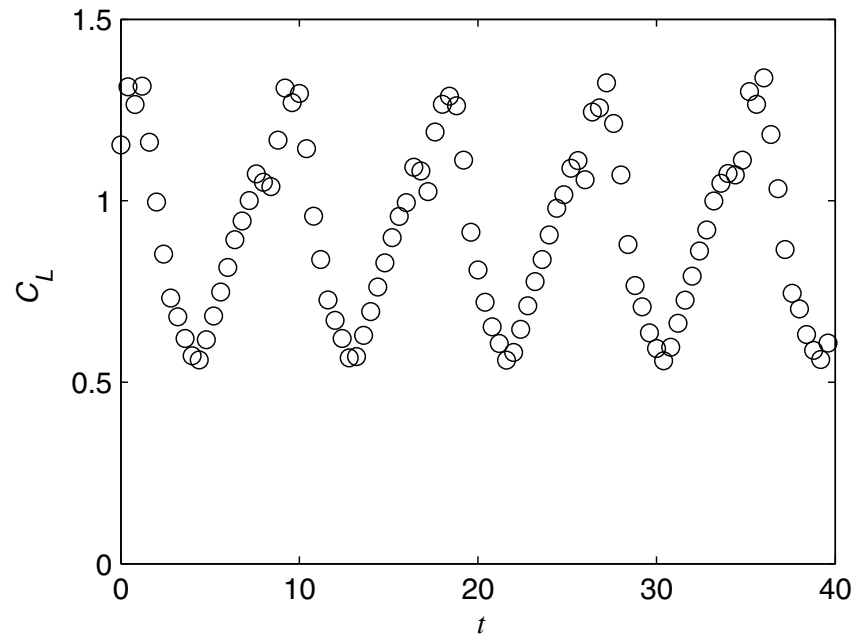

(b)

Figure 7. Time trace of lift coefficient for (a) NACA0012 at $\alpha=11^{\circ}$ and (b) E374 at $\alpha=13^{\circ}$.

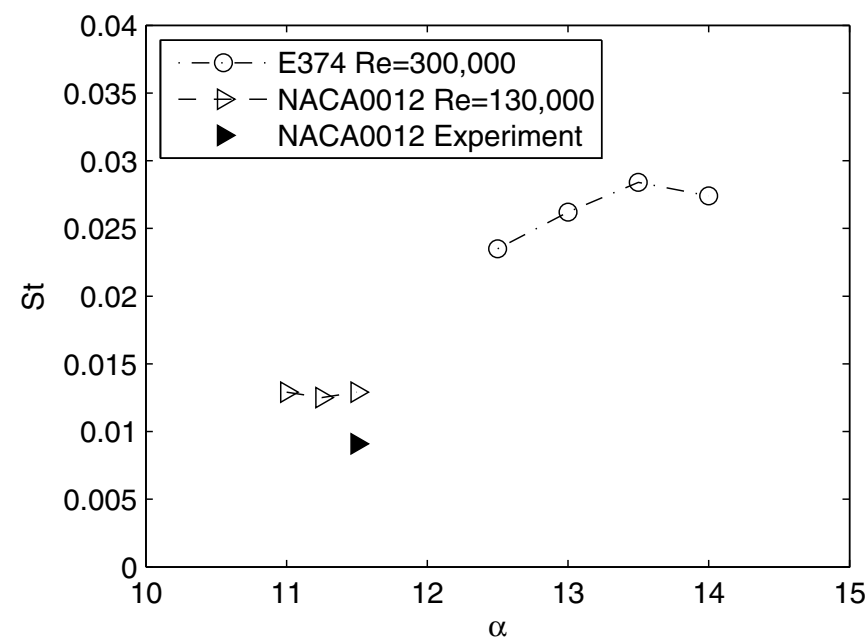

Figure 8. Variation of Strouhal number with incidence for high amplitude oscillations.

with a maximum $C_{L}=1.27$ (compared to $C_{L}=1.25$ in the experiment). The only discrepancy for this case appears to be the hysteresis loop, which spreads over a range of $3^{\circ}$ in the experiment but only $1^{\circ}$ in the model (too small to be shown on Fig. 6(c), but clearly visible when comparing data from calculations with increasing versus decreasing incidence). For this case stall cells were observed experimentally, which may be active in preventing the flow from reattaching as the incidence is reduced from a stalled configuration and leading to a larger hysteresis loop. The main purpose in presenting this case, however, is to demonstrate that for some aerofoils the model predicts no oscillations during stall, confirming that the low-frequency oscillations are not a general result of the modelling strategy, but are driven by the relevant flow phenomena. The aerofoil geometry is crucial is determining whether there will be a low frequency oscillation during stall.

One final point to note is the general robustness of the model for angles beyond the stall. Even though one should not expect the data to be meaningful (the boundary-layer assumption having long since been violated) it is still possible to get what are apparently quite sensible results, a feature that may well make this modelling approach useful for application to dynamic stall.

\subsection{Nature of the flow oscillations near stall}

In this section we consider in more detail the nature of the flow oscillations seen near stall. Figure 7 shows a plot of the developed oscillation in the lift coefficient for the NACA0012 aerofoil at $\alpha=$ $11^{\circ}$ and the E374 aerofoil at $\alpha=13^{\circ}$. For both cases the lift coefficient shows a regular oscillation in time. Near the peak of lift coefficient there is some evidence of vortex shedding, but the variations due to shedding are an order of magnitude less than the peak-to-peak amplitude of the low-frequency oscillation The period 14.8 seen for the NACA0012 corresponds to $S t=0.0129$, while the period 8.6 for the E374 corresponds to $S t=0.0262$. The weak variation of St with incidence is shown on Fig. 8. For the NACA0012 a value of $S t=$ 0.0091 can be deduced from the experiments ${ }^{(21)}$. Both experiment and simulation for the NACA0012 show values of St that are even lower than the 0.017 to 0.03 range observed previously ${ }^{(19)}$. This suggests that the shape of the aerofoil plays a dominant role, since Bragg et $a l^{(19)}$ showed that the variation with of St with Re was small, over the range of Re for which the low frequency oscillation occurs.

The structure of the burst cycle can be studied by plotting flow properties at various points in the cycle. We identify a phase $\phi=0^{\circ}$ with the maximum lift coefficient. Figure 9 shows $C_{p}, \delta^{*}$ and $H$ at four phases $\left(\phi=0^{\circ}, 90^{\circ}, 180^{\circ}\right.$ and $\left.270^{\circ}\right)$ during the cycle. At $\phi=0^{\circ}$ the flow has just become separated over the whole of the upper surface. As $\phi$ increases the pressure coefficient flattens out, the displacement thickness grows and by $\phi=90^{\circ}$ the flow is fully stalled. This process has however reduced the adverse pressure gradient in the leading edge region, with the result that the flow can reattach to form a separation bubble. At $\phi=180^{\circ}$ we see that we have a leading edge separation bubble with attached flow after the bubble up to approximately $x=0.4$. The bubble cannot hold itself in this position however, and as the pressure minimum grows the reattachment point moves rearwards due to the increasing adverse pressure gradient. This process can be seen by comparing $\phi=180^{\circ}$ with $\phi=270^{\circ}$. Eventually the entire flow is once again separated and the cycle repeats.

Figure 10 compares the model data with experimental measurements of Broeren \& Bragg ${ }^{(20)}$ for the turbulent reattachment (after a separation bubble) and the turbulent separation that occurs further 

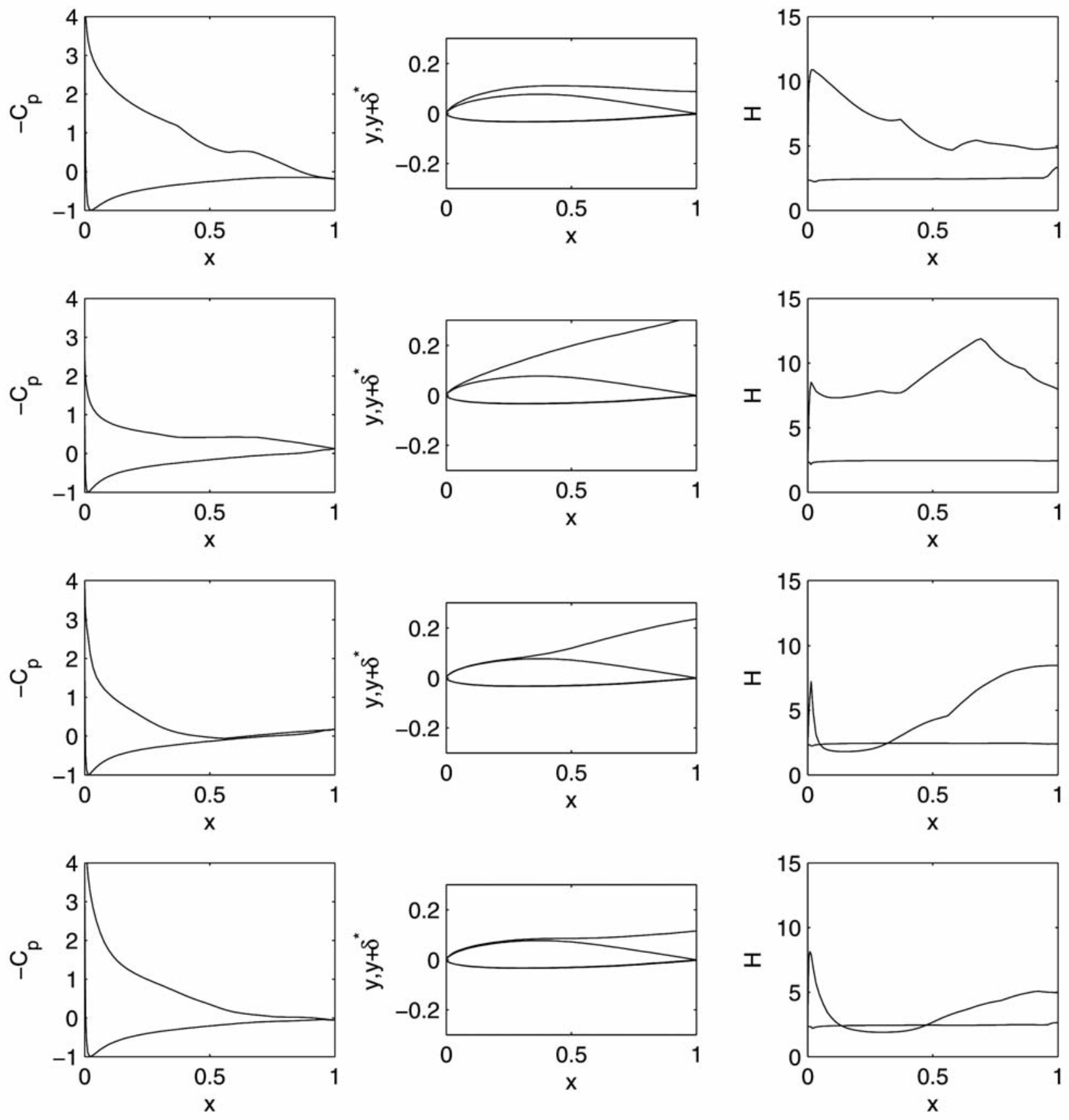

Figure 9. Variation of pressure coefficient (left), displacement thickness (centre) and shape factor (right) during the burst cycle for the E374 aerofoil. From the top the phase angles are $\phi=0^{\circ}$ (maximum lift), $90^{\circ}, 180^{\circ}$ and $270^{\circ}$.

downstream. At all phases during the cycle there is a laminar boundary-layer separation at $x=0$, followed by transition. For $100^{\circ}$ $<\phi<260^{\circ}$ there is a transitional separation bubble present that occupies less than $10 \%$ chord. The variation of the details is in good qualitative agreement with experiment. For example the rapid downstream movement of the reattachment point follows the downstream movement of the reattachment and the phase lag between these is in agreement. The most significant difference is the comparatively early turbulent separation seen in the model calculations, up to $20 \%$ chord earlier than in the experiments. Overall though, there do not appear to be any features from the experimental data that are not captured by the viscous-inviscid interaction model. 


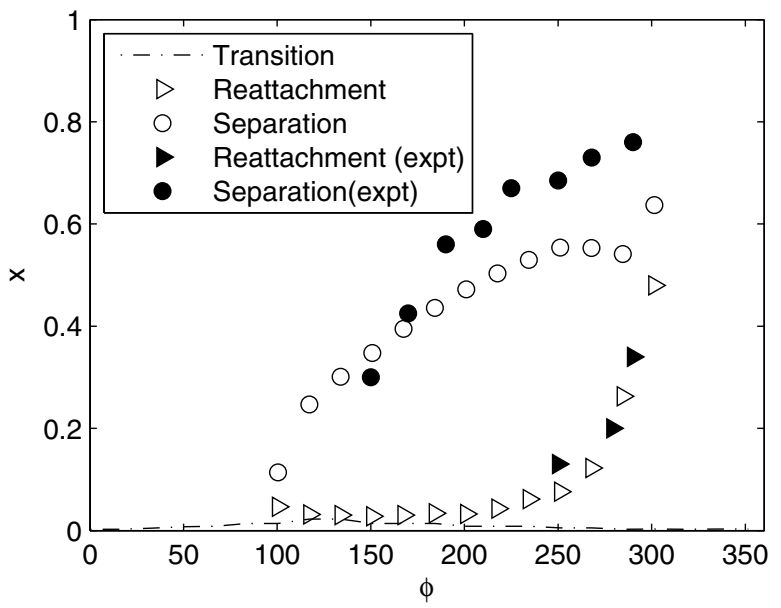

Figure 10. Variation of transition, reattachment and turbulent separation locations with phase during a bursting cycle, superimposed with experimental data from Broeren and $\mathrm{Bragg}^{(20)}$ for $\mathrm{E} 374$ at $\alpha=13^{\circ}$.

\subsection{DISCUSSION AND CONCLUSION}

The principal result of this paper is that the quite elaborate timedependent formation, growth and reformation of the separation bubble during a bursting cycle near stall can be predicted with an extension of standard viscous-inviscid interaction methods to unsteady flow. In particular this supports the conjecture of Gaster ${ }^{(17)}$ that bubble bursting occurs when there is a runaway effect involving the bubble displacement interacting with the potential flow. Details of transition and turbulence are contained within relatively simple models, which nevertheless appear to contain sufficient of the relevant flow physics (for example response of transition and reattachment points to a time-varying local pressure gradient) to model separation bubble phenomena. Detailed results from the model are in good agreement with published data, including the range of angles of incidence over which the high amplitude, lowfrequency motions are found, and the related Strouhal numbers.

In developing the unsteady model a number of steps have been taken which may be useful in other contexts:

1. A transport equation formulation has been proposed in Equations (1) and (3-5). For steady flow this reduces to standard forms for the transition and turbulence models, but for unsteady flow it allows laminar and turbulent flow zones to convect downstream and means that the important effects of flow history are included. In particular, because the amplification factor is treated in this way it is possible for boundary layers to relaminarise if there is no longer a strong local growth of disturbances. The need for such an extension of the approximate envelope method to unsteady flow was recently highlighted by Radespiel $e t a l^{(28)}$ and it should be relatively straightforward to couple the present model with a Reynolds-averaged code.

2. The detailed transition model has been revised, via Equation (7), to include the possibility that transition is self-sustained i.e. that transition will take place even if there are no upstream disturbances. The standard method precludes this, but evidence from direct simulations ${ }^{(24)}$ is that this sort of transition via an absolute instability in a separation bubble can occur on aerofoils.

Aspects of the modelling can no doubt be improved. The panel method and transport equations can be extended to higher order and coefficients in the laminar and transition models can be better tuned to aerofoil flow. Also the low Reynolds number performance of the turbulence model can probably be improved; typically the model gives smaller skin friction values after reattachment than are obtained in comparable direct numerical simulations. Nevertheless, the method as it stands is already suitable for application to other problems, including dynamic stall, flapping wings and flexible structures, as a cheap alternative to Reynolds-averaged methods.

\section{REFERENCES}

1. Jones, B.M. Stalling, J Aero Soc, 38, 1934, pp 753-770.

2. MCGregor, I. Regions of Localised Boundary Layer Separation and their Role in Nose Stalling of Aerofoils, 1954, PhD thesis, Queen Mary College, University of London.

3. Gaster, M. On the Stability of Parallel Flows and the Behaviour of Separation Bubbles, 1963, PhD thesis, Queen Mary College, University of London.

4. Horton, H.P. Laminar Separation Bubbles in Two and Three Dimensional Incompressible Flow, 1968, PhD thesis, Queen Mary College, University of London.

5. WoOdward, D.S. An Investigation of the Flow in Separation Bubbles, $1970 \mathrm{PhD}$ thesis, Queen Mary College, University of London.

6. Alam M. Direct Numerical Simulation of Laminar Separation Bubbles, 1999, PhD thesis, Queen Mary and Westfield College, University of London.

7. Young, A.D. and HorTon, H.P. Some results of investigations of separation bubbles, AGARD CP, No.4, 1966 pp 779-811.

8. Horton, H.P. A semi-empirical theory for the growth and bursting of laminar separation bubbles, 1963, ARC CP 1073.

9. RoBERTS, W.B. Calculation of laminar separation bubbles and their effect on aerofoil performance, , AIAA J, 1980, 18, (1), pp 25-31.

10. Weibust, E. Bertelrud, A and Ridder, S.O. Experimental investigation of laminar separation bubbles and comparison with theory, $J$ Aircr, 1987, 24, (5), pp 291-297.

11. Drela, M. \& GiLES, M.B. Viscous-inviscid analysis of transonic and low Reynolds number airfoils, AIAA J, 1987, 25, (10), pp 1347-1355.

12. Rist, U. AND MAUCHER, U. Investigations of time-growing instabilities in laminar separation bubbles, Eur J Mechanics B-Fluids, 21, (5), 2002 pp 495-509.

13. MarXen, O, Rist, U. and Wagner, S. Effect of spanwise-modulated disturbances on transition in a separated boundary layer, AIAA J, 42, (5), 2004, pp 937-944.

14. Alam, M. AND SAndham, N.D. Direct numerical simulation of 'short' laminar separation bubbles with turbulent reattachment, J Fluid Mech, 2000, 410, pp 1-28.

15. Spalart, P.R. and Strelets M.K. Mechanisms of transition and heat transfer in a separation bubble, J Fluid Mech, 2000, 403, pp 329-349.

16. Pauley, L.L., Moin, P. And Reynolds, W.C. The structure of twodimensional separation, J Fluid Mech, 220, 1990, pp 397-411.

17. GASTER, M. The structure and behaviour of laminar separation bubbles, ARC R\&M, 1969, 3595.

18. Zaman, K.B.M.Q, McKinzie, D.J, And Rumsey, C.L. A natural lowfrequency oscillation of the flow over an airfoil near stalling conditions, J Fluid Mech, 1989, 202, pp 403-442.

19. Bragg, M.B., Heinrich, D.C. And Khodadoust, A. Low-frequency flow oscillation over airfoils near stall, AIAA J, 1993, 31, (7), pp 1341-1343.

20. Broeren, A.P. AND BragG, M.B. Spanwise variation in the unsteady stalling flowfields of two-dimensional airfoil models, AIAA J, 2001, 39, (9), pp 1641-1651.

21. RinOIE, K AND TAKEMURA, N. Oscillating behaviour of laminar separation bubble formed on an aerofoil near stall, Aeronaut $J$, March 2004, pp 153-163.

22. Drela, M. XFOIL V6.96 available from http://web.mit.edu/drela/ Public/web/xfoil/

23. RosenheAd, L. Laminar Boundary Layers, 1988, Dover, New York.

24. Jones, L.L., SANDBERG, R.D. AND SANDHAM, N.D. Direct numerical simulations of forced and unforced separation bubbles on an airfoil at incidence, J Fluid Mech, 2008, 602, pp 175-207.

25. Katz, J and Plotkin, A. Low-Speed Aerodynamics, 2001, Second Edition, CUP.

26. GRAHAM, J.M.R. The lift on an airfoil in starting flow, J Fluid Mech, 1983, 133, pp 413-425.

27. Аввотt, I.H. And von Doenhoff, A.E. Theory of Wing Sections, 1959, Dover, New York, US.

28. Radespiel, R., Windte, J and Scholz, U. Numerical and experimental flow analysis of moving airfoils with laminar separation bubbles, AIAA $J, 2007,45$, (2), pp 1346-1356. 
29. Young, A.D. Boundary Layers, 1989, BSP, Oxford, UK.

30. Green, J.E., Weeks, D.J. and Brooman, J.W.F. Prediction of turbulent boundary layers and wakes in compressible flow by a lag-entrainment method, ARC R\&M, 1977, 3791.00

\section{APPENDIX A: DETAILS OF THE MODELLING}

\section{A.1 LAMINAR BOUNDARY LAYER}

An unsteady laminar boundary layer is modelled as a return-toequilibrium flow, in which spatially non-uniform velocity gradients drag the boundary layer from one equilibrium state towards another. A complete method is constructed based on Equation (3) with

$B_{H}=\frac{1 \cdot 25 U_{H}}{\operatorname{Re}_{\theta} \theta}\left(H_{e q}-H\right)$

where $\operatorname{Re}_{\theta}=U_{e} \theta / \mathrm{u}$ is the local momentum-thickness Reynolds number and $H_{e q}$ is given in terms of the usual pressure gradient parameter

$m=-\operatorname{Re} \theta^{2} \frac{\mathrm{d} U_{e}}{\mathrm{~d} s}$

as

$$
\begin{aligned}
H_{e q} & =2 \cdot 01+\frac{0.6}{1-9 \cdot 5 m} & & \text { for } m \leq 0.09 \\
& =6 \cdot 15+100(m-0.09) & & \text { for } m>0.09
\end{aligned}
$$

Coefficients in Equation (A.3) have been determined such that the method is comparable in performance to the widely-used Thwaites $\operatorname{method}^{(29)}$. In particular the zero pressure gradient and stagnation point solutions are identical to Thwaites and separation occurs at $\mathrm{m}$ $=0.09$. For $H>4$ an additional term $1 \cdot 2 U_{H} m / \theta$ is added to Equation (A.1). The action of this is mainly cosmetic, ensuring that strong separation regions have a strong local effect, flattening the pressure distribution in the dead-air region of separation bubbles. The friction term in the momentum equation follows directly from a fit to tabulated data for Thwaites method ${ }^{(29)}$

$$
\begin{aligned}
& \frac{\tau_{w}}{\rho}=\frac{U_{e}}{\operatorname{Re} \theta}(-0 \cdot 17+0 \cdot 67 \exp (-0 \cdot 89(H-2))) \text { for } H \geq 2 \\
& =0.5 \frac{U_{e}}{\operatorname{Re} \theta} \quad \text { for } H<2 .
\end{aligned}
$$

It should be noted that the current method extends beyond separation, with $H$ allowed to grow indefinitely so long as $m$ is strongly positive, while $\tau_{w} / \rho$ will converge to $-0 \cdot 17$. Laminar reattachment is also possible if the pressure gradient parameter drops below $m=0.09$.

\section{A.2 LAMINAR-TURBULENT TRANSITION}

A suitable transition prediction scheme for integral calculations can be found in Drela and Giles ${ }^{(11)}$. In the present notation the right hand side of Equation (4) for this basic model of convective instability (CI) is given by

$$
B_{n}=0 \quad \text { for } \operatorname{Re}_{\theta}<10^{a}
$$

where the critical Reynolds number $\operatorname{Re}_{c r i t}=10^{a}$ is found using $a=\left(\frac{1 \cdot 415}{H-1}-0 \cdot 489\right) \operatorname{Tanh}\left(\frac{20}{H-1}-12 \cdot 9\right)+\frac{3 \cdot 295}{H-1}+0 \cdot 44$

while for $\operatorname{Re}_{\theta} \geq 10^{a}$ we have

$B_{n}=\frac{0 \cdot 3 U_{e} f_{1}\left(f_{2}+f_{3}\right)}{2 \theta}$

with subsidiary functions given by

$$
\begin{aligned}
& f_{1}=0 \cdot 01\left((2 \cdot 4 H-3 \cdot 7+2 \cdot 5 \operatorname{Tanh}(1 \cdot 5 H-4 \cdot 65))^{2}+0 \cdot 25\right)^{0.5} \ldots \\
& f_{2}=\frac{6 \cdot 54 H-14 \cdot 07}{H^{2}} \\
& f_{3}=\frac{0 \cdot 058(H-4)^{2}}{H-1}-0 \cdot 068
\end{aligned}
$$

The method was devised to match Falkner-Skan (i.e. equilibrium) boundary layer stability characteristics. It should be noted that boundary layers computed according to the present laminar boundary model will not correspond exactly to equivalent equilibrium flows. However, in the absence of a comparable $e^{n}$ method calibrated to aerofoil boundary layers, we will use the method given by Equations (4, 6 and A.5-A.10) above.

\section{A.3 TURBULENT FLOW}

For turbulent flow we choose the Green et $a l^{(30)}$ lag-entrainment model. In the present implementation the method involves the solution of transport equations for the shape factor $H$ and the entrainment factor $E$. In the notation from Section 2.1 we have

$B_{H}=\frac{U_{H}\left(E-H_{1}\left(0 \cdot 5 C_{f}-(1+H) \Lambda\right)\right)}{\theta \mathrm{d} H_{1} / \mathrm{d} H}$
$B_{E}=\frac{U_{E} f_{E}}{\theta}\left(\frac{2 \cdot 8\left(\Omega_{e q}^{1 / 2}-\lambda \Omega^{1 / 2}\right)}{H_{1}+H}+\Lambda_{e q}-\Lambda\right)$

where $C_{f}=\tau_{w} /\left(1 / 2 \rho U_{e}^{2}\right)$ is the skin friction coefficient and $\Lambda$ is a pressure gradient parameter defined by

$$
\Lambda=\frac{\theta}{U_{e}} \frac{\mathrm{d} U_{e}}{\mathrm{~d} s}
$$

Subsidiary functions are given by

$H_{1}=3 \cdot 15+\frac{1 \cdot 72}{H-1}-0 \cdot 01(H-1)^{2}$

$\frac{\mathrm{d} H_{1}}{\mathrm{~d} H}=-\frac{1 \cdot 72}{(H-1)^{2}}-0 \cdot 02(H-1)$

$C_{f}=C_{f_{0}}\left(\frac{0.9}{H\left(1-6 \cdot 55 \sqrt{0 \cdot 5 C_{f_{0}}}\right)-0.4}-0.5\right)$

$C_{f_{0}}=\frac{0 \cdot 01013}{\log _{10} \operatorname{Re}_{\theta}-1 \cdot 02}-0 \cdot 00075$ 


$$
\begin{aligned}
& \Lambda_{e q}=\frac{1}{H+1}\left(0 \cdot 5 C_{f}-\frac{1}{H_{1}}\left(\frac{\Omega_{e q} \lambda^{-2}-0 \cdot 32 C_{f_{0}}}{1 \cdot 2}\right)^{0.5}\right) \\
& E_{e q}=H_{1}\left(0 \cdot 03 \frac{\left(H^{2}-1\right)(H-1)}{H^{3}}-\frac{C_{f}(5+H)}{8 H}\right) \\
& \Omega=0 \cdot 32 C_{f_{0}}+0 \cdot 024 E+1 \cdot 2 E^{2} \\
& \Omega_{e q}=0 \cdot 32 C_{f_{0}}+0 \cdot 024 E_{e q}+1 \cdot 2 E_{e q}^{2}
\end{aligned}
$$$$
f_{E}=\frac{E(E+0 \cdot 02)+0 \cdot 2667 C_{f_{0}}}{E+0 \cdot 01}
$$

Corrections to the model due to secondary effects are usually included via the parameter $\lambda$. Only the surface curvature effect concerns us here, with the correction factor being

$$
\lambda=1+\beta \frac{2}{3} \frac{\theta}{R}\left(H+H_{1}\right)\left(\frac{H_{1}}{H}+0 \cdot 3\right)
$$

... (A20) with $R$ the radius of curvature (positive for convex curvature) and $\beta$ $=7$ for positive $R$ and $\beta=4.5$ for negative $R$. The model was validated by Green et al against the 1968 Stanford conference data with results agreeing at least as well as the best differential methods tested at the time of that conference. 